\title{
402. Sonographische Akutdiagnostik beim stumpfen Bauch- und Thoraxtrauma
}

\author{
M. Aufschnaiter und H. Kofler \\ II. Universitätsklinik für Chirurgie, Anichstraße 35, A-6020 Innsbruck
}

\section{Sonographic Acute Diagnosis in Blunt Abdominal and Thoracic Trauma}

Summary. 204 cases of blunt abdominal or thoracic trauma are presented, which were investigated by sonography for severe intra-abdominal or intrathoracic hemorrhage demanding urgent surgical intervention. In 202 patients bleeding of this kind was accurately diagnosed or excluded. Two cases were not evaluable. Thus peritoneal lavage was necessary in only 2 of 204 cases. The possibilities and limitations of the two techniques are compared.

Key words: Sonography - Intra-abdominal intrathoracic bleeding - Peritoneal lavage.

Zusammenfassung. Es wird über 204 Fälle von stumpfen Bauch- und Thoraxtraumen berichtet, die mittels Sonographie auf das Vorliegen einer dringlich operationsbedürftigen Blutung im Thorax und Abdomen untersucht wurden. 202mal wurde korrekt eine klinisch relevante Blutung nachgewiesen bzw. ausgeschlossen. 2 mal konnte keine Aussage gemacht werden, so daß die Peritoneallavage lediglich in 2 von 204 Fällen durchgeführt werden mußte. Möglichkeiten und Grenzen von Sonographie und Peritoneallavage werden einander gegenübergestellt.

Schliusselwörter: Ultraschalldiagnostik - Stumpfes Bauchtrauma - Stumpfes Thoraxtrauma.

\section{Die Aussagekraft der Sonographie nach stumpfem Bauchtrauma}

\author{
K. H. Hauenstein ${ }^{1}$, B. Wimmer ${ }^{1}$, D. Zavisic ${ }^{2}$ und E. Farthmann ${ }^{2}$ \\ ${ }^{1}$ Institut für Röntgendiagnostik der Universitätsklinik Freiburg, \\ ${ }^{2}$ Chirurgische Universitätsklinik Freiburg, Hugstetter Straße 55, D-7800 Freiburg
}

\section{Diagnostic Value of Ultrasonography in Blunt Abdominal Trauma}

Summary. Following blunt abdominal trauma ultrasonographic evaluation allows rapid and reliable detection of intraperitoneal fluid accumulation indicating organ laceration. Modern movable equipment allows an immediate examination in the shock room. In $27 \%$ of 779 patients examined since 1980 pathological findings were detected; in 92 patients the sonographic findings indicated the necessity for immediate surgery. The rate of false diagnoses was about $1 \%$. To correlate free intraperitoneal blood volume and sonographic findings we performed sonography in patients undergoing peritoneal dialysis. Special attention was paid to minor fluid collections beneath the liver, which are detectable but do not necessitate immediate surgery.

Key words: Sonography - Blunt abdominal trauma.

Zusammenfassung. Nach stumpfem Bauchtrauma kann die Sonographie schnell und sicher intraperitoneale Flüssigkeit als Hinweis auf eine Organruptur nachweisen. Die modernen transportablen Geräte ermöglichen die Untersuchung auch im Schockraum. Von 779 seit 1980 untersuchten Patienten zeigten 27\% pathologische Befunde; bei 92 Patienten war eine sofortige Operation nötig. Die Fehlerquote lag bei $1 \%$. Um eine Korrelation zwischen intraperitonealer Blutmenge und sonographischem Befund herstellen zu können, haben wir Peritonealdialysepatienten nach Gabe definierter Flüssigkeitsmengen über den liegenden Dialysekatheter untersucht. Besonders interessierten nur schmale Flüssigkeitsansammlungen subhepatisch, da hier chirurgischerseits unter sonographischen Verlaufskontrollen abgewartet werden kann.

Schliisselwörter: Sonographie - Stumpfes Bauchtrauma. 